\title{
FATORES QUE AFETAM O CONSUMO ALIMENTAR E A NUTRIÇÃO DO IDOSO
}

\section{FACTORS THAT AFFECT THE AGED PEOPLE FOOD INTAKE AND NUTRITION}

\author{
Maria Teresa Fialho de Sousa CAMPOS \\ Josefina Bressan Resende MONTEIRO' \\ Ana Paula Rodrigues de Castro ORNELAS²
}

RESUMO

\begin{abstract}
Os fatores que afetam o consumo alimentar das pessoas idosas são reconhecidos como de risco para o desenvolvimento da má nutrição. Este artigo busca fazer uma revisão abrangente sobre esses fatores, procurando também elucidar as condições de nutrição dos idosos brasileiros. A compreensão desses fatores pelos profissionais de saúde amplia o entendimento das condições peculiares que determinam o estado nutricional do idoso e que devem ser trabalhadas de forma interligada, para manutenção ou restauração da eutrofia.
\end{abstract}

Termos de indexação: idoso, consumo de alimentos, nutrição.

\section{ABSTRACT}

The factors that affect the aged people food intake are recognized as risk for the development of undernourishment. This article aims at providing a general review about these factors, trying to elucidate the conditions of the Brazilian aged people nutrition. The understanding of these factors by the professionals of health enlarges the knowledge of the peculiar conditions which determine the aged people nutritional status, and which should be worked in an interactive way, for the maintenance or restoration of the normal status.

Index terms: aged, food consumption, nutrition.

\section{INTRODUÇÃO}

A distribuição etária da população mundial tem apresentado visível alteração nas últimas décadas, em razão da expansão da expectativa de vida e do conseqüente aumento de idosos, o que representa novos desafios no campo da pesquisa nutricional.

O Brasil, à semelhança dos demais países latino-americanos, está passando por um processo de envelhecimento rápido e intenso. A evolução da população geriátrica brasileira constitui um grande desafio, enquanto a população brasileira crescerá 3,22 vezes até o ano 2025 , o segmento acima de 65 anos aumentará 8,9 vezes, e o acima de 80 anos, 15,6 vezes. Com isso, a proporção de idosos que em 1980 era menor que 6\%, subirá em menos de 50 anos, para 14\% devendo o Brasil ocupar o sexto lugar na esfera mundial no ano 2 025, com uma estimativa de 31,8 milhões. Mantendo-se a tendência demográfica atual, em números absolutos, o país terá uma das maiores

\footnotetext{
${ }^{(1)}$ Departamento de Nutrição e Saúde, Universidade Federal de Viçosa. Av. P.H. Rolfs, s/n, Campus, 36571-000,Viçosa, MG, Brasil. Correspondência para/Correspondence to: M.T.F.S.CAMPOS.E-mail: mtcampos@mail.ufv.br

${ }^{(2)}$ Nutricionista, Mestranda em Microbiologia Agrícola, Universidade Federal de Viçosa.
} 
populações de idosos do mundo (Kalache et al., 1987; Ramos et al., 1987; Cançado, 1992; Ramos et al.,1993; Veras, 1994; Chaimowicz, 1997; Chaimowicz, 1998; Moriguti et al.,1998).

O envelhecimento, apesar de ser um processo natural, submete o organismo a diversas alterações anatômicas e funcionais, com repercussões nas condições de saúde e nutrição do idoso. Muitas dessas mudanças são progressivas, ocasionando efetivas reduções na capacidade funcional, desde a sensibilidade para os gostos primários até os processos metabólicos do organismo (Amerine et al., 1965; Mitchell et al., 1978; Watkin, 1982; Wenck et al., 1983).

Associado às alterações decorrentes do envelhecimento, é freqüente o uso de múltiplos medicamentos que influenciam na ingestão de alimentos, na digestão, na absorção e na utilização de diversos nutrientes, o que pode comprometer o estado de saúde e a necessidade nutricional do indivíduo idoso (Marucci, 1993; Najas et al.,1994).

Nesse sentido, várias pesquisas (Brown et al., 1977; O' Hanlon \& Kohrs, 1978; Stiedemann et al., 1978; Vir \& Love, 1979; Arhontaki, 1990) têm demonstrado deficiência de energia, vitaminas e minerais em pessoas, acima de 65 anos, que residem em asilos ou domicílios, fato atribuído aos fatores socioeconômicos e às doenças presentes, além de alterações no modo de vida e nos hábitos alimentares.

Este artigo objetiva evidenciar os fatores que afetam o consumo alimentar de idosos, bem como caracterizar as condições da nutrição geriátrica no Brasil, às vésperas da virada do século. A compreensão desses fatores é uma alternativa viável para auxiliar os profissionais de saúde a reverter os índices de má nutrição na geriatria, o que resultaria em melhores condições de saúde e, conseqüentemente, de vida.

\section{Estado nutricional da população idosa brasileira}

Em 1989, foi realizada a Pesquisa Nacional sobre Saúde e Nutrição (PNSN), com o objetivo central de avaliar o estado nutricional da população brasileira mediante a coleta de dados antropométricos. Essa pesquisa, de âmbito nacional, revelou que a situação nutricional de adultos e idosos sofreu grande alteração, nos últimos 15 anos. Estima-se uma redução de $36 \%$ no grupo de baixo peso, com aumento maior dos casos de sobrepeso e obesidade, tendo reduzido o número de indivíduos antropometricamente normais. Essa tendência foi verificada tanto no meio rural quanto no urbano, em todas as faixas etárias. Portanto, a população adulta e idosa brasileira apresenta alta prevalência de baixo peso e também de obesidade.

No que tange a geriatria, no início da década de 90, a freqüência do baixo peso atingia 20,75\% dos homens e $17,00 \%$ das mulheres. Em números absolutos, o país possuía 1 milhão e 300 mil idosos com baixo peso. Os idosos de baixa renda eram os mais atingidos, visto que, à medida que aumentava a renda per capita, reduzia o percentual de baixo peso. O sobrepeso e, principalmente, a obesidade afetava, proporcionalmente, mais as mulheres do que os homens. Praticamente, metade da população idosa brasileira possuía excesso de peso, em todas as regiões do Brasil (Coitinho et al., 1991).

Estudos realizados em regiões metropolitanas no Brasil têm demonstrado um aumento do excesso de peso na população idosa, em ambos os sexos (Chaar, 1996; Frank, 1996; Pereira, 1998).

A elevada prevalência de desvio nutricional na população idosa vem sendo demonstrada por meio de diferentes estudos, em vários países, onde, a desnutrição, o sobrepeso e a obesidade predominam sobre os indivíduos eutróficos. Esses resultados são decorrentes das condições peculiares em que os idosos se encontram, seja no ambiente familiar, vivendo sozinho, ou em residência de Terceira Idade, agravadas pelas condições socioeconômicas, pelas alterações fisiológicas inerentes à idade e pela progressiva incapacidade para realizar sozinho as suas atividades cotidianas. Nesse contexto, os efeitos da alimentação inadequada, tanto por excesso como por déficit de nutrientes, têm expressiva representação, o que reflete num quadro latente de má nutrição em maior ou menor grau (Campos, 1996).

A situação nutricional da população geriátrica brasileira sinaliza a necessidade de buscar conhecer e compreender todas as peculiaridades que afetam o consumo alimentar do idoso, levando-se em consideração as características regionais nas quais estão inseridos.

\section{Fatores que afetam o consumo de nutrientes nos idosos}

Os idosos apresentam condições peculiares que condicionam o seu estado nutricional. Alguns desses condicionantes são devidos às alterações fisiológicas próprias do envelhecimento, enquanto outros são influenciados pelas enfermidades presentes e por fatores relacionados com a situação socioeconômica e familiar (Nogués, 1995).

\section{Fatores socioeconômicos}

Entre os fatores mais importantes na gênese da má nutrição do ancião, encontram-se os externos, como os fatores psicossociais, tais como perda do cônjuge, depressão, isolamento social, pobreza, integração social, capacidade de deslocamento, capacidade cognitiva e outros associados à própria enfermidade.

Estima-se que mais de $15 \%$ dos anciãos têm alimentação diária com menos de 1000 kcal/dia, 
aumentando esta porcentagem entre as populações menos favorecidas economicamente. Grande parte dos idosos consome alimentos de menor custo, em virtude dos insuficientes recursos econômicos provenientes de aposentadorias e, ou, pensões (Nogués, 1995).

No Brasil, uma das características marcantes da população idosa é o baixo poder aquisitivo, situação que é agravada em razão da exclusão dos idosos do mercado de trabalho (Veras, 1994), fato que, certamente, resulta na aquisição de alimentos de custos mais acessíveis e contribui para a monotonia da alimentação.

Conforme Aranceta-Bartrina (1988), a integração social é outro fator que tem papel relevante na alteração do consumo alimentar do idoso. A solidão familiar e social predispõe o idoso à falta de ilusão e preocupação consigo, fazendo com que se alimente de forma inadequada em quantidade e qualidade. Nesses casos, há tendência ao desestímulo para comprar e preparar alimentos variados e nutritivos (Arhontaki, 1990; Moriguti et al., 1998). Verifica-se, com freqüência, elevado consumo de produtos industrializados, como doces e massas, ou de fácil preparo, como chás e torradas. Essa modificação no comportamento alimentar certamente afeta a adequação de nutrientes ao organismo dos idosos e coloca-os em risco de má nutrição (Aranceta-Bartrina, 1988; Arhontaki, 1990; Nogués, 1995).

O estado de ânimo do idoso para ingerir o alimento é, muitas vezes, modificado por atitudes simples, como posicionar-se confortavelmente à mesa em companhia de outras pessoas. Os levantamentos que comparam participantes idosos de Programa de Alimentação com não participantes mostraram que o fornecimento de energia, e a ingestão de proteína, vitaminas e minerais pelo primeiro grupo foi aumentado (Podrabsky, 1995). Isso indica que a integração realmente tem conotação social muito importante na alimentação do idoso e influencia na aceitação ou na recusa do alimento (Nogués, 1995).

De acordo com Morales-Rodriguez et al. (1989), a má nutrição do idoso pode também ser decorrente de sua progressiva incapacidade de realizar sozinho as atividades cotidianas. A coordenação motora, geralmente, é comprometida e tende a piorar com as doenças neurológicas, o que pode levá-lo a evitar alimentos que possam causar dificuldades de manipulação durante a refeição, o que contribui para a inadequação alimentar (Moriguti et al. , 1998). Nessas circunstâncias, a aquisição de alimentos e a preparação das refeições podem tornar tarefas muito difíceis (Podrabsky, 1995).

As questões concernentes à capacidade funcional e à autonomia do idoso podem ser mais importantes que a própria questão da morbidade, pois se relacionam, diretamente, com a qualidade de vida. Os inquéritos domiciliares, realizados em três capitais do Brasil, demonstraram proporção crescente de indivíduos que necessitam de auxílio para realizar atividades diárias, como transferir-se da cama para o sofá, vestir-se, alimentar-se e cuidar da própria higiene, dependência esta que é intensificada com o avanço da idade (Chaimowicz, 1998). Outro inquérito domiciliar brasileiro revelou que pouco mais da metade dos idosos entrevistados podia realizar todas as atividades diárias sem nenhuma necessidade de ajuda (Ramos et al., 1993). O primeiro estudo epidemiológico longitudinal com idosos residentes na comunidade, no Brasil, revelou que o perfil da população não diferiu de estudos transversais anteriores, mostrando maioria de mulheres, viúvas, vivendo em domicílios multigeracionais, com alta prevalência de doenças crônicas, distúrbios psiquiátricos e incapacidade físicas (Ramos et al., 1998). Exatamente na velhice, fase da vida em que os indivíduos necessitam de maior apoio familiar e comunitário, verifica-se que no país está ocorrendo redução desta assistência. A situação de isolamento vivido pelo idoso brasileiro é ainda potencializada pela sua posição econômica, que, nos últimos anos, tem contribuído para o aumento da desnutrição (Coitinho et al., 1991).

$\mathrm{Na}$ terceira idade, deve-se também estar atento a outros fatores, tais como perda do cônjuge e depressão, pois ambos levam à perda do apetite ou à recusa do alimento. Por outro lado, a ansiedade pode desencadear o aumento excessivo de peso.

\section{Alterações fisiológicas}

$\mathrm{Na}$ velhice ocorrem mudanças progressivas no organismo, o que conduz a efetivas reduções nas funções fisiológicas (Watkin, 1982; Wenck et al., 1983; Quintero-Molina, 1993).

As mudanças fisiológicas que interferem no estado nutricional são: diminuição do metabolismo basal, redistribuição da massa corporal, alterações no funcionamento digestivo, alterações na percepção sensorial e diminuição da sensibilidade à sede. Com exceção das duas primeiras, todas as outras podem interferir, diretamente, no consumo alimentar (Quintero-Molina, 1993; Nogués, 1995).

\section{Alterações no funcionamento do aparelho digestivo}

Conforme Russel (1992), é importante compreender os efeitos da idade no trato gastrintestinal, pois as mudanças podem afetar a necessidade nutricional da população idosa, bem como a sua dose de necessidade de medicamentos.

Há numerosos estudos sobre a diminuição da função gastrintestinal em virtude do avanço da idade, mas pouco se conhece acerca das alterações morfológicas. Na maioria dos casos, o funcionamento gastrintestinal mantém-se relativamente íntegro, porque há grande capacidade funcional no intestino, no pâncreas e no fígado. As mudanças clinicamente relevantes neste sistema, em humanos, incluem decréscimo no limiar do gosto; atrofia 
da mucosa gástrica, conseqüentemente menor produção de ácido clorídrico, diminuição do fator intrínseco e menor absorção da vitamina $\mathrm{B}_{12}$; e decréscimo no tamanho do fígado (Russel, 1992; Nogués, 1995).

\section{Alterações na percepção sensorial}

As alterações sensoriais podem estar associadas ao decréscimo do apetite nas pessoas idosas (Rolls,1992). Essas mudanças abrangem declínio e eventual perda da acuidade visual, audição, olfato e sensação de gustação (Rivlin, 1981; Chernoff, 1987; Podrabsky, 1995). Estas alterações são parciais e afetam o comportamento alimentar do idoso, conforme Cooper citado por Arhontaki (1990). Dentre todas as mudanças sensoriais, o olfato e a gustação interferem mais diretamente na ingestão de alimentos (Chernoff, 1987; Jurdi-Haldeman, 1988; Nogués, 1995), e de acordo com Moriguti (1998), a visão prejudicada também a influencia negativamente. Não há dúvida que o apetite no idoso é influenciado, principalmente, pela palatabilidade dos alimentos (Rolls, 1992).

$\mathrm{Na}$ terceira idade, um dos fatores mais relevantes na diminuição do consumo alimentar é a redução da sensibilidade por gostos primários doce, amargo, ácido e salgado (Rolls, 1992; Nogués, 1995; Shuman, 1998). O gosto tem sua base anatômica no número de gemas gustativas das papilas linguais. Nos jovens, este número corresponde a mais de 250 para cada papila, enquanto as pessoas acima de 70 anos têm menos de 100 (Nogués, 1995), o que vem endossar a colocação de Russel (1992) sobre o decréscimo do limiar de detecção e identificação do "flavor" em conseqüência do envelhecimento. Isso significa que o idoso necessita de maior concentração do sabor atribuído ao alimento, em comparação com adultos jovens. Estudos recentes demonstram que a dificuldade que o idoso possui para detectar o sabor doce dos alimentos o predispõe a adoçar mais os alimentos (Nogués, 1995), comportamento similar ocorre com relação ao sabor salgado. O idoso tende a concentrar o tempero dos alimentos para ajustá-lo ao paladar, que está alterado.

Outro fator que exerce importante função na sensibilidade aos sabores é o sentido olfativo (Guyton, 1977). Os quimiorreceptores olfativos, localizados na parte interna do nariz, são responsáveis pela sensação de percepção do estímulo causado por substâncias voláteis. De acordo com Rolls (1992) e Nogués (1995), com a idade, ocorre diminuição da percepção olfativa. No entanto, ainda existem poucos relatos associados às alterações decorrentes na geriatria (Russel, 1992). Para este autor, a forma como o alimento é identificado pelo idoso está sob a influência de diversos fatores bastante comuns na geriatria, como utilização de medicamentos, estado nutricional, higiene oral, estado do sistema nervoso central e hábito de fumar.

A visão prejudicada é outro fator também comum nas pessoas idosas, o que pode levar à diminuição do apetite, em decorrência da diminuição do reconhecimento dos alimentos e da habilidade de alimentar-se.

Nesse sentido, os hábitos alimentares do indivíduo idoso são muito influenciados pelas alterações sensoriais, o que reflete um quadro latente de anorexia em maior ou menor grau, dependendo da intensidade.

\section{Alterações na capacidade mastigatória}

A mastigação é importante para uma boa nutrição no idoso (Nagao, 1992). Com o envelhecimento, os hábitos de mastigação mudam, acentuadamente, tanto nos homens como nas mulheres. Essas alterações na capacidade mastigatória do idoso são devidas ao aparecimento freqüente de cáries e doenças periodontais; às próteses totais ou parciais inadaptadas ou em péssimo estado de conservação, e à ausência de dentes. Esses fatores interferem no comportamento inicial do processo digestivo, favorecendo sua inadequação tanto no aspecto enzimático como no mecânico (Nogués, 1995; Hayflick, 1996).

Atualmente, sabe-se que o edentulismo não é conseqüência natural do envelhecimento, e que os dentes naturais, quando bem tratados, podem permanecer em funcionamento por toda a vida (Dunkerson, 1998). As principais causas de ausência de dentes e de uso de próteses totais na terceira idade são decorrentes de cáries não tratadas e da periodontite, embora essas causas possam ser previníveis com a tecnologia atual. A perda de apetite em idosos tem sido, geralmente, relacionada com ausência de elementos dentários e com o uso de próteses, de acordo com Carlsson citado por Cormack (1998). As pessoas que usam dentaduras mastigam 75 a $85 \%$ menos eficientemente que aquelas com dentes naturais, o que leva à diminuição do consumo de carnes, frutas e vegetais frescos, razão porque idosos com próteses totais tendem a consumir alimentos macios, facilmente mastigáveis, pobres em fibras, vitaminas e minerais, fato que pode ocasionar consumo inadequado de energia, ferro e vitaminas (Shuman,1998).

\section{Alterações na composição e no fluxo salivar e na mucosa oral}

As células das glândulas salivares são reduzidas em número nas pessoas idosas (Scott, 1977a,b), embora não tenha ainda sido demonstrada nenhuma ligação entre o envelhecimento e a redução da secreção salivar espontânea ou estimulada (Heft \& Baum, 1984; Baum, 1989). Segundo Hayflick (1996), o fluxo da saliva e de seus componentes permanecem estáveis durante todo o processo de envelhecimento.

A saliva tem papel relevante no processo digestivo, na prevenção de cáries e das doenças periodontais e na lubrificação das mucosas. Nas pessoas idosas, os estudos que sugerem diminuição da função salivar têm sido 
confundidos com a presença de enfermidades e, ou, com uso de medicamentos (Russel, 1992). De acordo com este autor, é provável que as poucas células salivares que sobrarem em uma idade mais avançada sejam mais eficientes em suas funções. De fato, muitas medicações têm o poder de afetar a função da glândula salivar e tornar-se mais intenso o ressecamento bucal, segundo Lewis et al. citado por Cormack (1998). O consumo médio de medicamentos por idosos é em torno de 1,7 tipos diferentes, e o fluxo salivar diminui em relação direta com o aumento no número de medicações, com efeitos potencialmente hipossalivatórios. A maior parte dessas drogas está associada a efeitos de inibição do fluxo salivar, resultando num potencial aumento da suscetibilidade a cárie dental (Cormack, 1998). Dessa forma, a xerostomia apesar de afetar mais de $70 \%$ dos idosos e afetar, significativamente, a ingestão de alimentos, pode ser conseqüência do abuso de medicamentos nos gerontes. Este fato suscita algumas afirmativas, como a de Dunkerson (1998), de que a xerostomia não é conseqüência natural do envelhecimento, mas geralmente decorrente do uso de medicamentos e de outras doenças sistêmicas.

Outros autores, como Nogués (1995), enfatizam a elevada incidência de cáries, principalmente as de superfícies radiculares, e de doenças periodontais no idoso, em razão da precariedade da higiene bucal, do alto acúmulo de carboidratos fermentáveis e das alterações salivares que condicionam uma espessa capa de mucina, potencializando essas doenças. Há dificuldade em realizar a higiene bucal por causa da perda gradual de movimentos coordenados (Dunkerson, 1997).

Com o avanço da idade, as modificações mais evidentes na mucosa oral são proeminência das glândulas sebáceas, aparência lisa na superfície da mucosa e diminuição da espessura do epitélio bucal e lingual. Dentre essas mudanças, a aparência lisa na mucosa e a diminuição da espessura do epitélio na cavidade oral interferem diretamente no consumo de alimentos, diminuindo o apetite. Essa perda de apetite pode ser decorrente do aumento da sensibilidade na mucosa oral, o que ocasiona sensação de ardor no idoso, quando este ingere alimentos quentes ou frios.

\section{Alterações na estrutura e função do esôfago}

Disfunções clinicamente significantes do esôfago são raras em todos os grupos de idade, embora leves mudanças tenham sido descritas com o envelhecimento. Isto inclui diminuição na amplitude das contrações e no número de ondas peristálticas após a deglutição do alimento e aumento no número de desordens nas contrações do corpo do esôfago (Hollis \& Castell, 1974; Castell, 1988; Russel, 1992).

Regularmente, enfermidades neurológicas relacionadas com a idade resultam em significante disfunção secundária do esôfago e podem causar pneumonia por aspiração e má nutrição. Mas, em geral, mesmo na velhice, a função esofagiana é bem preservada (Hosoda, 1992; Russel, 1992).

\section{Alterações na estrutura e função do estômago e intestino}

A atrofia da mucosa gástrica no idoso resulta na menor produção de ácido clorídrico e na diminuição na secreção do fator intrínseco, provocando menor absorção da vitamina $B_{12}$, com conseqüente instalação da anemia perniciosa. Esse tipo de anemia ocorre somente quando o idoso apresenta grave atrofia da mucosa gástrica. O estômago com gastrite leve ou moderada continua a secretar fator intrínseco suficiente para prevenir a má absorção da vitamina $B_{12}$, pelo mecanismo normal (Suter et al., 1991; Russell 1992; Nogués, 1995).

Segundo Podrabsky (1995) e Nogués (1995), a hipocloridria afeta bastante a absorção de cálcio e de ferro não-heme. O ácido clorídrico mantém tanto o ferro férrico quanto o cálcio solúveis por meio dos seus efeitos acidificantes para serem absorvidos no trato intestinal. Nos casos em que há baixa produção de ácido clorídrico, a absorção desses nutrientes é diminuída em razão de sua insolubilidade em pH acima de 5,0. Apesar de o ácido ascórbico aumentar a solubilidade e a absorção do ferro ferroso em pH neutro ou levemente alcalino, a quelação entre estes só ocorrerá num meio ácido. Com relação ao cálcio, as evidências recentes mostram que apenas a ingestão isolada deste apresenta limitada biodisponibilidade na falta da acidez gástrica (Russell, 1992).

A ocorrência da gastrite no ancião está, dentre outros fatores, associada ao microorganismo Hélicobacter pylori. Estima-se que $80 \%$ das pessoas acima de 60 anos que tenham leve gastrite apresentem anticorpos ao Hélicobacter pylori no seu sangue, em comparação com $50 \%$ daqueles que não têm a doença (Faisal et al., 1990).

O envelhecimento afeta, também, o esvaziamento gástrico de uma refeição, tornando-o mais lento. Este fato foi evidenciado em um estudo, em que as pessoas idosas gastaram aproximadamente 123 minutos para promover $\mathrm{o}$ esvaziamento gástrico da metade do bolo alimentar, contra 50 minutos nos adultos jovens (Evans et al., 1981).

Modificações intestinais também são observadas no indivíduo idoso, ocorrendo certo grau de atrofia na mucosa e no revestimento muscular que resulta na deficiência de absorção de nutrientes e favorece a instalação de diverticulose, em virtude da menor motilidade no intestino grosso e cólon (Geokas \& Haverback, 1969; Nogués, 1995; Podrabsky, 1995). Essas alterações na motilidade também contribuem para o aparecimento da constipação, que é freqüente na geriatria. No entanto, esse quadro de constipação no idoso pode ainda estar relacionado com a baixa ingestão de líquidos e de fibras, com o menor número de refeições por dia, com a depressão 
e com a inatividade, de acordo com Sullivan \& Walls citados por Shuman (1998).

No que concerne aos nutrientes, a ênfase tem sido na diminuição da absorção de cálcio no intestino, com a idade em ambos os sexos, devido, provavelmente, às alterações nos vários processos de transporte (Shuman,1998).

O crescimento bacteriano excessivo no intestino pode também ocorrer, como resultado da diminuição da secreção ácida. Tal fato interfere na disponibilidade biológica dos nutrientes e pode resultar na menor ação dos sais biliares, na má absorção da gordura e na diarréia (Podrabsky, 1995).

\section{Alterações no pâncreas}

A função pancreática diminui em ratos mais velhos quando comparados com os novos (Greenberg \& Holt, 1986). Em pessoas idosas, parece que o pâncreas é capaz de funcionar bem, sem condições de estresse, no entanto, sob estimulação repetida com secretina ou colecistocinina, a secreção pancreática da referida população cai significativamente (Gullo et al., 1986).

A preservação da função pancreática também foi confirmada por Arora et al. (1989), em idosos com até 91 anos. Os resultados mostraram que, durante 24 horas, a excreção fecal de gorduras, em uma dieta contendo $100 \mathrm{~g}$ deste nutriente por dia, foi em torno de 2,8 g para ambos os grupos de idade: 19 a 44 anos e 70 a 91 anos. Porém, quando o conteúdo de gordura na dieta foi aumentado para valores entre 115 a 120 g por dia, desenvolveram uma leve esteatorréia. Situação similar encontra-se nas dietas que contêm 1,5 g de proteína por kg de peso corpóreo diário. A excreção fecal de nitrogênio ficou equiparada entre os jovens e velhos, quando foi fornecido 1,0 g de proteína por kg de peso corpóreo (Werner \& Hambraeus, 1972).

Os efeitos das dietas ricas e pobres em fibras, 35 e $10 \mathrm{~g}$ por dia, respectivamente, nas taxas de excreção de gordura fecal no idoso foram também similares. Dentro dessa faixa de ingestão de fibras parece que o seu fornecimento não contribui para esteatorréia (Dutta \& Hlasko, 1985).

Resultados de estudos relativos à digestão e absorção de carboidratos vêm sinalizando uma diminuição na capacidade com a idade. Porém, a elevada prevalência de má digestão ou de absorção do carboidrato na velhice pode ser resultante da exposição deste nutriente à colônia de bactérias presente na flora intestinal (Feibusch \& Holt, 1982).

\section{Alterações na estrutura e na função do fígado e vias biliares}

Com o envelhecimento, o fígado é submetido a algumas alterações anatômicas e funcionais. As alterações anatômicas compreendem diminuição do peso do fígado e do número de hepatócitos e aumento de tecido fibroso. Conseqüentemente, em nível citoplasmático têm-se registrado mudanças que interferem na biotransformação dos fármacos, da síntese protéica, do metabolismo lipo-protéico e da secreção da bile, como também na redução da tolerância a outros agentes normalmente excretados por esse órgão (Geokas \& Haverback, 1969; Nogués, 1995).

De acordo com Shamburek \& Farrar (1990), a prevalência de pedras biliares aumenta com a idade. Estudos realizados em jejum e com contrações estimuladas de colecistocinina no plasma foram maiores nos indivíduos idosos, quando comparados com jovens, mas o volume da vesícula biliar e a taxa de esvaziamento obtiveram resultados similares. Parece que a sensibilidade da vesícula biliar pode estar diminuída nas pessoas idosas (Khalil et al., 1985).

\section{Diminuição da sensibilidade à sede}

O estado de hidratação é outro fator de extrema relevância em geriatria. No idoso a desidratação torna-se freqüente podendo desencadear outras doenças como enfermidades infecciosas e cerebrovasculares, que, neste último caso, muitas vezes, apresenta-se como um quadro de delirium (Nogués, 1995; Moriguti et al., 1998).

Sabe-se que a osmolaridade sérica mantém-se com a ingestão de líquidos e com a excreção renal de solutos. De acordo com Moriguti et al. (1998), a sua regulação depende da sintonia entre a capacidade renal de concentrar e diluir a urina e a ingestão de água motivada pela sede. Deve-se considerar que a ingestão de líquidos depende dos fatores ambientais, psicológicos e fisiológicos, e que a capacidade de concentração renal diminui com a idade (Nogués, 1995; Cormack, 1998; Moriguti et al., 1998).

A alteração na sensação de sede é atribuída à disfunção cerebral e, ou, à diminuição da sensibilidade dos osmorreceptores. No entanto, a menor ingestão de líquidos pode ainda ser decorrente de alguma debilidade física, pois, neste caso, existe certa dependência de outras pessoas. Este quadro de hipodipsia é agravado pela administração de diuréticos e de laxativos, muito freqüente nos idosos. Pouco consumo de água pelos idosos associada ao uso freqüente de diuréticos e laxantes, leva à desidratação (Nogués, 1995; Cormack, 1998; Moriguti et al., 1998)

\section{Efeitos secundários dos fármacos}

Os idosos sofrem com mais freqüência que os jovens efeitos adversos dos medicamentos. Isto é conseqüência da queda de suas funções vitais, da múltipla e simultânea medicação e de seu estado nutricional, 
muitas vezes deficiente nesta fase da vida. Os efeitos metabólicos e digestivos adversos, que alguns medicamentos de uso habitual em geriatria produzem, devem ser considerados na análise da ingestão de alimentos (Larralde, 1994; Nogués, 1995; Oliveira, 1999). Os mais freqüentes são:

- Tranqüilizantes e psicofármacos: favorecem o relaxamento e diminuem a absorção intestinal;

- Diuréticos e laxantes: ocasionam desidratação e depleção de eletrólitos como magnésio, potássio e zinco;

- Antibióticos: alteram a absorção intestinal por destruição da flora. Provocam má absorção de carboidratos, vitamina $B_{12}$, cálcio, ferro, magnésio e cobre e inibem a síntese protéica;

- Glicocorticóides: predispõem à gastrite, osteoporose (interferem na absorção do cálcio) e hiperglicemia, e

- Analgésicos: favorecem as gastrites e úlceras.

A terceira idade, em geral, apresenta multiplicidade de doenças, e portanto, consome maior número de medicamentos. O uso de diferentes medicamentos, nesta época da vida, tem deixado de ser esporádico para converter em habitual. A polifarmácia em idosos aumenta a incidência de efeitos colaterais e interações medicamentosas e o seu uso inadequado, freqüentemente, provoca complicações graves. Nesse sentido, a utilização, a longo prazo, de drogas terapêuticas que interferem na digestão, na absorção e no metabolismo de nutrientes pode, também, ocasionar desnutrição nos idosos, além de desenvolver anorexia (Fujita, 1992; Marucci, 1993; Larralde, 1994; Podrabsky, 1995; Marucci \& Gomes, 1997; Chaimowicz, 1998; Moriguti et al., 1998).

\section{CONSIDERAÇÕES FINAIS}

O aumento de pessoas idosas é um processo irreversível e que não pode ser negligenciado pela Ciência da Nutrição.

Nesse sentido, estudos sobre o consumo alimentar do idoso não devem se restringir à análise qualitativa e quantitativa. Na realização do planejamento dietético alimentar, é imprescindível a compreensão de todas as peculiaridades inerentes às mudanças fisiológicas naturais do envelhecimento, da análise dos fatores econômicos, psicossociais e de intercorrências farmacológicas associadas às múltiplas doenças que interferem no consumo alimentar e, sobretudo, na necessidade de nutrientes.

Atitudes simples, como servir as refeições em local agradável (limpo, arejado, de preferência de cor clara, com piso não-derrapante, com mobiliário adequado e com espaço livre para facilitar a circulação das pessoas), sentar o idoso confortavelmente à mesa em companhia de outras pessoas (familiares, amigos, dentre outras pessoas), disciplinar e fracionar o consumo de alimentos estabelecendo horários (oferecendo refeições menos volumosas mais vezes ao dia), oferecer a eles refeições atrativas (combinar, de acordo com as recomendações para a faixa etária, alimentos construtores, energéticos e reguladores, oferecendo refeições coloridas) e saborosas (usar temperos naturais como alho, cebola, cebolinha, cheiro verde, salsa, orégano e outros, evitando, assim, o abuso do sal), e promover um contraste de cor entre os utensílios e o forro da mesa, melhoram o estado de ânimo do idoso, influenciando, positivamente, o seu apetite. Pode-se, também, colocar um fundo sonoro neste ambiente, desde que a opção seja por músicas suaves e que atendam à preferência da faixa etária, pois o idoso tende a degustar os alimentos com mais tranqüilidade. Tais condutas proporcionam ao idoso mais prazer com a alimentação.

A adoção dessas condutas, associada ao domínio cognitivo dos fatores que afetam o consumo alimentar dos idosos, propiciará aos profissionais de saúde e as casas de amparo a Terceira Idade o investimento em intervenções que contribuirão, positivamente, para o consumo alimentar desse segmento populacional e, conseqüentemente, auxiliarão na melhoria do seu estado nutricional.

\section{REFERÊNCIAS BIBLIOGRÁFICAS}

AMERINE, M.A., PANGBORN, R.M., ROESSLER, E.B. Principles of sensory of food. New York: Academic Press, 1965. 602p.

ARANCETA-BARTRINA, J. Pautas dietéticas nutricionales para la vejez. Nutrición Clínica, v.8, n.6, p.34-40, 1988.

ARHONTAKI, J. Desenvolvimento e avaliação de formulações para alimentação de idosos. Viçosa, 1990. 99p. Dissertação (Mestrado em Ciência e Tecnologia de Alimentos) - Universidade Federal de Viçosa, 1990.

ARORA, S., KASSARJIAN, Z., KRASINSKI, S.D. Effect of age on tests of intestinal and hepatic function in the healthy humans. Gastroenterology, Philadelphia, v.96, n.6, p.1560-1565, 1989.

BAUM, B.J. Salivary gland fluid secretion during aging. Journal of the American Geriatrics Society, New York, v.37, n.5, p.453-458, 1989.

BROWN, P.T., BERGAN, J.G., PARSONS, E.P. Dietary status of elderly people. Journal of the American Dietetic Association, Chicago, v.71, n.7, p.41-45, 1977.

CAMPOS, M.T.F.S. Efeitos da suplementação alimentar em idosos. Viçosa, 1996. 119p. Dissertação (Mestrado em Ciência e Tecnologia de Alimentos) - Universidade Federal de Viçosa, 1996.

CANÇADO, F.A.X. Transformações nos padrões de mortalidade por idade e causas. In: SEMINÁRIO NACIONAL DE ESPECIALISTAS MULTIDISCIPLINARES EM TERCEIRA IDADE: A POPULAÇÃO IDOSA NO BRASIL, 1., Belo Horizonte, 1992. Anais... Belo Horizonte : Fundação João Pinheiro, 1992. p.85-126.

CASTELL, D.O. Effects of aging on gastrointestinal physiology. Practical Gastroenterology, v.12, p.28-37, 1988. 
CHAAR, C. F.S. Avaliação nutricional e alimentar de idosos matriculados do Centro de saúde. Revista da Área de Ciências Biológicas e da Saúde, v.1, n.1, p.12-15, 1996.

CHAIMOWICZ, F. A saúde dos idosos brasileiros às vésperas do século XXI: problemas, projeções e alternativas. Revista de Saúde Pública, São Paulo, v.31, n.2, p.184-200, 1997.

CHAIMOWICZ, F. Os idosos brasileiros no século XXI: demografia, saúde e sociedade. Belo horizonte : Postgraduate, 1998, p.5-92.

CHERNOFF, R. Aging and nutrition. Nutrition Today, Annapolis, v.6, n.2, p.4-11, 1987.

COITINHO, D.C., LEÃO, M.M., RECINE, E., SICHIERI, R. Pesquisa nacional sobre saúde e nutrição: condições nutricionais da população brasileira - adulto e idoso. Brasília : INAN, 1991. p.39.

CORMACK, E. A saúde oral do idoso. [online] p.1-10, 1998. Available from www: <www.odontologia.com.br/artigos/ geriatria.html >.

DUNKERSON, J.A. A odontologia na terceira idade. [online] p.1-11, 1997. Available from www: <www. odontologia.com.br/artigos/ geriatria.html>.

DUNKERSON, J.A. O atendimento ao paciente odontogeriátrico. p.1-9, 1998. Available from [online] www: <www. odontologia.com.br/ artigos/geriatria.html>.

DUTTA, S.K., HLASKO, J. Dietary fiber on pancreatic disease: effect of high fiber diet on fat malabsorption in pancreatic insufficiency and in vitro study of the interaction of dietary fiber with pancreatic enzymes. American Journal of Clinical Nutrition, Bethesda, v.41, n.3, p.517-526, 1985.

EVANS, M.A., TRIGGS, E.J., CHEUNG, M. Gastric emptying rate in the elderly: implications for drug therapy. Journal of the American Geriatrics Society, New York, v.29, n.5, p.201-205, 1981.

FAISAL, M.A., RUSSEL, R.M., SAMLOFF, I.M. Helicobacter pylori infection and atrophic gastritis in the elderly. Gastroenterology, Philadelphia, v.99, n.5, p.1543-1544, 1990.

FEIBUSCH, J.M., HOLT, P.R. Impaired absorptive capacity for carbohydrate in the aging human. Digestive Diseases and Sciences, New York, v.27, n.12, part 2, p.1095-1100, 1982.

FRANK, A.A. Estudo antropométrico e dietético de idosos. Rio de Janeiro, 1996. 156p. Dissertação (Mestrado em Nutrição Humana) - Universidade Federal do Rio de Janeiro, 1996.

FUJITA, Y. Nutritional requirements of the elderly: a japanese view. Nutrition Reviews, New York, v.50, n.12, p.449-453, 1992.

GEOKAS, M.C., HAVERBACK, B.J. The aging gastrintestinal tract. American Journal of Surgery, Newton, v.117, n.1, p.881-892, 1969.

GREENBERG, R.E., HOLT, P.R. Influence of aging upon pancreatic digetive enzymes. Digestive Diseases and Sciences, New York, v.31, n.9, p.970-977, 1986.

GULLO, L., VENTRUCCI, M., NALDONI, P. Aging and exocrine pancreatic function. Journal of the American Geriatrics Society, New York, v.34, n.11, p.790-792, 1986.

GUYTON, A.C. Tratado de fisiologia médica. 5.ed. Rio de Janeiro : Interamericana, 1977. 1037p.
HAYFLICK, L. Como e porque envelhecemos. Rio de Janeiro : Campus, 1996. 366p.

HEFT, M.W., BAUM, B.J. Unstimulated and stimulated parotid salivary flow rate in individuals of different ages. Journal of Dental Research, Washington DC, v.63, n.10, p.1182-1185, 1984.

HOLLIS, J.B., CASTELL, D.O. Esophageal function in elderly men. Annals Internal Medicine, Philadelphia, v.80, n.3, p.371-374, 1974.

HOSODA, S. The gastrintestinal tract and nutrition in the aging process: an overview. Nutrition Reviews, New York, v.50, n.12, p.372-373, 1992.

JURDI-HALDEMAN, D., NAPIER, A.K. Perceived relationships between taste and smell acuity and food intake in the elderly. Topics in Clinical Nutrition, v.3, n.4, p.4-8, 1988.

KALACHE, A., VERAS, R.P., RAMOS, L.R. O envelhecimento da população mundial: um desafio novo. Revista de Saúde Pública, São Paulo, v.21, n.3, p.200-210, 1987.

KHALIL, T., WALKER, J.P., WIENER, I. Effect of aging on gallbladder contraction and release of cholecystokinin-33 in humans. Surgery, St. Louis, v.98, n.3, p.423-429, 1985.

LARRALDE, J. Nutrición en el anciano. In: HERNÁNDEZ, J.A.M. Nutrición, dieta y salud. [s.l: s.n], 1994. p.69-85.

MARUCCI, M.F.N., GOMES, M.M.B.C. Interações droga-nutriente em idosos. In: PAPALÉO NETTO, M. Gerontologia. São Paulo: Atheneu, 1997. p.273-283.

MARUCCI, M.F.N. Equilíbrio nutricional na terceira idade. In: CONGRESSO NACIONAL, ALIMENTOS E EQUILÍBRIO NUTRICIONAL: PERSPECTIVAS PARA O SÉCULO XXI, 3., São Paulo, 1993. Anais... São Paulo: SBAN, 1993. p.35-36.

MITCHELL, H.S., RYNBERGEN, H.J., ANDERSON, L. Nutrição em geriatria. In: MITCHELL, H.S. Nutrição. 16.ed. Rio de Janeiro : Interamericana, 1978. Cap. 19: p.271-278.

MORALES-RODRIGUEZ, P., GONZÁLES-REIMERS, E., SANTOLARIA FERNANDEZ, F. Relación entre involución psicofisica del anciano y su estado nutricional. Nutrición Hospitalaria, Madrid, v.4, n.5, p.276-282, 1989.

MORIGUTI, J.C. et al. Nutrição no idoso. In: OLIVEIRA, J.E., MARCHINI, J.S. Ciências nutricionais. São Paulo : Sarvier, 1998. Cap.14: p.239-251.

NAGAO, M. The effects of aging on mastication. Nutrition Reviews, New York, v.50, n.12, p.434-437, 1992.

NAJAS, M.S., ANDREZZA, R., SOUZA, A.L.M., SACHS, A., GUEDES, L.R.S., RAMOS, L.R., TUDISCO, E.S. Padrão alimentar de idosos de diferentes estratos sócio-econômicos residentes em localidade urbana da Região Sudeste, Brasil. Revista de Saúde Pública, São Paulo, v.28, n.3, p.187-191, 1994.

NOGUÉS, R. Factors que afectan la ingesta de nutrientes en el anciano y que condicionan su correcta nutrición. Nutrición Clínica, v.15, n.2, p.39-44, 1995.

O'HANLON, P., KOHRS, M.B. Dietary studies of older americans. American Journal of Clinical Nutrition, Bethesda, v.31, n.6, p.1257-1269, 1978.

OLIVEIRA, R.B. Farmacologia aplicada ao paciente geriátrico. Revista Médica de Minas Gerais, Belo Horizonte, v.9, n.1, p.25-27, 1999. 
PEREIRA, R.A. Avaliação antropométrica do estado nutricional. In: SICHIERI, R. Epidemiologia da obesidade. Rio de Janeiro : UERJ, 1998. Cap.4: p.43-63.

PODRABSKY, M. Nutrição e envelhecimento. In: MAHAN, K.L., ARLIN, M.T. Krause, alimentos, nutrição e dietoterapia. Rio de Janeiro : Roca, 1995. Cap.14: p.255-269.

QUINTERO-MOLINA, R. Nutrición en los ancianos. Geriatrika, v.9, n.1, p.14-18, 1993.

RAMOS, L.R., VERAS, R.P., KALACHE, A. Envelhecimento populacional: uma realidade brasileira. Revista de Saúde Pública, São Paulo, v.21, n.3, p.211-224. 1987.

RAMOS, L.R., ROSA, T.E.C., OLIVEIRA, Z.M., MEDINA, M.C.G., SANTOS, F.R.G. Perfil do idoso em área metropolitana na Região Sudeste do Brasil: resultados de inquérito domiciliar. Revista de Saúde Pública, São Paulo, v.27, n.2, p.87-94, 1993.

RAMOS, L.R. Estudo de seguimento por dois anos de idosos residentes em São Paulo, Brasil: metodologia e resultados preliminares. Revista de Saúde Pública, São Paulo, v.32, n.5, p.397-407, 1998.

RIVLIN, R.S. Nutrition and aging: some unanswered questions. American Journal of Medicine, Newton, v.71, n.3, p.337-340, 1981.

ROLLS, B.J. Aging and appetite. Nutrition Reviews, New York, v.50, n.12, p.422-426, 1992.

RUSSEL, R.M. Changes in gastrintestinal function attributed to aging. American Journal of Clinical Nutrition, Bethesda, v.55, n.6, p.1203S-1207S, 1992. Supplement.

SCOTT, J. Degenerative changes in the histology of the human submandibular salivary glands. Journal Biologie Buccale, v.5, p.311-319, 1977a.

SCOTT, J. Quantitative age changes in the histological structure of human submandibular salivary glands. Archives of Oral Biology, Oxford, v.22, n.3, p.221-227, 1977b.
SHAMBUREK, R.D., FARRAR, J.T. Disorders of the digestive system in the elderly. New England Journal of Medicine, Boston, v.322, n.7, part 1, p.438-443, 1990.

SHUMAN, J.M. Nutrição no envelhecimento. In: MAHAN, L.K., STUMP, S.E. Alimentos, nutrição e dietoterapia. 9.ed. São Paulo : Roca, 1998. Cap. 14: p.293-312.

STIEDEMANN, M., JANSEN, C., HARRIL, I. Nutritional status or elderly man and woman. Journal of the American Dietetic Association, Chicago, v.73, n.2, p.132-138, 1978.

SUTER, P.M., GOLNER, B.B., GOLDIN, B.R., MORROW, F.D., RUSSELL, R.M. Reserval of protein: bound vitamin $B_{12}$ malabsorption with antibiotics in atrophic gastrits. Gastroenterology, Philadelphia, v.101, n.4, p.1039-1045, 1991.

VERAS, R.P. País jovem com cabelos brancos: a saúde do idoso no Brasil. Rio de Janeiro : Relume Dumará, 1994. p.9-224.

VIR, S.C., LOVE, A.H.G. Nutritional status of institutionalized and non institutionalized aged in Belfast, Northern Ireland. American Journal of Clinical Nutrition, Bethesda, v.32, n.9, part 3, p.1934-1947, 1979.

WATKIN, D.M. The physiology of aging. American Journal of Clinical Nutrition, Bethesda, v.36, n.4, p.750-758, 1982.

WENCK, D.A., BAREN, M., DEWAN, S.P. Nutrition: the challeng of being well nourished. 2.ed. New Jersey : [s.n.], 1983. Chapter 17: p.399-409.

WERNER, I., HAMBRAEUS, L. The digestive capacity of elderly people. In: CARLSON, P. Nutrition in old age. Uppsala, Sweden : Almquist and Wicksell, 1972. p.55-60.

Recebido para publicação em 7 de maio de 1999 e aceito em 18 de janeiro de 2000. 